\title{
Desarrollo de un pay de harina de frijol negro San Luis con mermelada de chilacayote de altas propiedades nutricionales
}

\author{
Development of a San Luis black bean flour pay, with chilacayote \\ jam of high nutritional properties
}

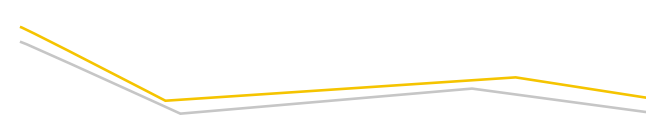

Esteban Misael Zumaran-Alvarado*; Manuel Juárez-García*四, José
Mancillas-Medina*, Martha Ávila-Ontiveros*, Areli Leyva-Maldonado*

Zumaran-Alvarado, E. M., Juárez-García, M., Mancillas-Medina, J., Ávila-Ontiveros, M., \& Leyva-Maldonado, A. (2017). Desarrollo de un pay de harina de frijol negro San Luis con mermelada de chilacayote de altas propiedades nutricionales. Investigación y Ciencia de la Universidad Autónoma de Aguascalientes, 25(71), 27-33.

RESUMEN

Se realizó un estudio para evaluar un pay a partir de harina de frijol con mermelada de chilacayote. Consistió en el desarrollo de cuatro formulaciones a partir de la sustitución parcial de harina de frijol por una de trigo, de 50:50 (T1), 65:35 (T2), 70:30 (T3) y 75:25 (T4), en mayor proporción la de trigo. Los resultados indicaron por medio de una evaluación sensorial la mejor formulación de acuerdo a los panelistas (T4). A su vez, se compararon el pay experimental y el comercial por medio de una prueba $t$ de Student y se observaron diferencias significativas $(p<0.05)$. El pay experimental (PE) presentó valores en proteína de $6.3 \%$, el comercial (PC) $2.5 \%$ y en fibra, el PE $0.9 \%$ por $0.3 \%$ del PC. Esto indica un producto altamente nutritivo y una alternativa de valor agregado del grano en el estado de Zacatecas.

\section{ABSTRACI}

A study was conducted to evaluate a pay from bean flour with chilacayote jam. This was the development

Palabras clave: pay; harina; frijol; evaluación; nutritivo; experimental.

Keywords: pay; flour; beans; evaluation; nutritious; experimental.

\section{Recibido: 9 de agosto de 2016, aceptado: 13 de junio de 2017}

* Ingeniería en Industrias Alimentarias, Instituto Tecnológico Superior Zacatecas Norte. González Ortega 3, Centro, C. P. 98400, Río Grande, Zacatecas, México. Correo electrónico: misaelza@hotmail.com; juareztec2011@hotmail.com; jdmancillas@yahoo.com;

mavilaontiveros@gmail,com; arelilm@hotmail.com

$凶$

Autor para correspondencia of four formulations from the partial substitution of bean flour wheat flour, these were 50:50 (T1), 65:35 (T2), 70:30 (T3) and 75:25 (T4), with higher proportion of wheat flour. The results indicated through sensory evaluation the best formulation according to panelists (T4). In turn, this was compared with the experimental pay under the trade through a Student $t$ test and significant differences were observed ( $p$ $<0.05)$. The experimental pay (PE) protein showed values in $6.3 \%$ and $2.5 \%$ for commercial pay (PC), and for fiber PE $0.9 \%$ by $0.3 \%$ for PC. This indicates a highly nutritious product and an value-added grain alternative in the state of Zacatecas.

\section{INTRODUCCIÓN}

En la actualidad, los estudios referentes al frijol (Phaseolus vulgaris L.) son de suma importancia debido a sus propiedades benéficas y a que se cultiva prácticamente en todo el mundo (EcuRed, 2015; Voysest, 2000). La piel del frijol tiene gran cantidad de flavonoides con poder antioxidante. Los principales componentes del grano son: proteínas (16-30\%), fibra (3-8\%), glúcidos (55-65\%), lípidos (1$5 \%)$ y minerales (3-5\%) (Rodiño, 2000). De manera adicional a estos compuestos posee elevada cantidad de calcio, hierro y vitaminas tales como la tiamina y el ácido fólico. Dentro del contenido de proteínas del frijol destaca la abundancia de lisina y poco contenido de aminoácidos azufrados (metionina y cisteína) (Bello Pérez, 2006; Ulloa, Rosas Ulloa, Ramírez Ramírez, \& Ulloa Rangel, 2011).

Dentro del grupo de leguminosas de grano para consumo humano podemos considerar a las 
IIVESTIGACIÓn Y CIERCIA DE LA UNIVERSIDAD AUTÓNOMA

habichuelas, frijoles, porotos, alubias, habas y judías como una de las más importantes, ya que su área de cultivo se extiende por los cinco continentes y desde el punto de vista nutricional es una fuente económica de proteínas para la dieta de muchos pueblos (Champ, 2002; Flórez, 2007; Galván-Moreno, \& Sagahón-Canales, 2014; Granito, Guinand, Pérez, \& Pérez, 2009; Longoria-Cordero \& Rivera-Acosta, 2015; Márquez-Rodríguez y López-Aguilar, 2014; Ulloa et al., 2011).

De acuerdo con la Organización de las Naciones Unidas para la Agricultura y la Alimentación (FAO, 2013) la producción mundial de frijol en el año 2009 fue de 1, 041, 349.90 millones de t; en 2010 aumentó $9.94 \%$, en 2011 hubo un decremento de $45.48 \%$, durante los años 2012 y 2013 incrementó $19.56 \%$ la producción del frijol y en 2014 fue de 1, 273, 957 t, con un incremento de $22.3 \%$ (SIAP, 2015).

De acuerdo con datos de la FAO el principal productor de frijol a nivel mundial es Brasil, al cierre de 2010 mostró una participación de 16\%, 6 en el total de la producción mundial; siguen India (15.9\%), Myanmar (10.5\%), China (8.9\%) y México (5.8\%) (FIRA, 2015; SAGARPA, 2010). De acuerdo con la superficie sembrada a nivel nacional destacan los estados de Zacatecas (33.6\%), Durango (15.1\%), Sinaloa (6.9\%), Chihuahua (7\%) y Chiapas (6.5\%). Asimismo, en el estado de Zacatecas se siembran 596,944 ha, de las cuales se cosechan 541,484, con una producción estimada de 355,882 † y un rendimiento por hectárea de 0.66 t/ha. De las mismas, los distritos de Río Grande, Fresnillo, Zacatecas y Ojo Caliente aportan $98.4 \%$, en donde destaca el primero como principal productor de esta leguminosa con un total de 243,481 t, ya que contribuye con $68.4 \%$ de la producción estatal (Longoria-Cordero, \& Rivera-Acosta, 2015; SIAP, 2015).

Sin embargo, los productores en México enfrentan diversos problemas, como altos costos de producción y un deterioro continuo de los precios reales del producto (Ayala-Garay, SchwentesiusRindermann, \& Almaguer-Vargas, 2008). Asimismo, SAGARPA (2010) estimó que el consumo nacional de frijol en 2008 se ubicó en 1.2 millones de t. De esta forma, el consumo per cápita de frijol se recuperó en 2008 con respecto a 2007, alcanzó 11 kg al año por persona (FIRA, 2015). El frijol puede servir como un alimento funcional porque contiene una serie de compuestos bioactivos que pueden desempeñar un papel metabólico en los seres humanos (Paredes-C., Becerra-V., \& Tay-U., 2009). De la misma manera, se ha demostrado en diversos estudios que el consumo de frijol disminuye el riesgo de problemas de salud tales como la obesidad, diabetes, enfermedades del corazón incluso el cáncer (Gálvez \& Salinas, 2015).

Por otra parte, el chilacayote (Cucurbita ficifolia) es un fruto esférico que generalmente se consume como verdura y se usa para preparar dulces regionales, los frutos maduros se utilizan como forraje para ganado y aves de corral (FAO, 2013). También se le puede utilizar como mermelada. En producción nacional de chilacayote destacan el Estado de México (86\%) y Morelos (14\%) (SIAP, 2015). Los principales componentes del chilacayote son carbohidratos con $6.9 \%$, fibra $0.6 \%$, grasas $0.5 \%$ y los principales minerales que contiene son calcio y hierro (FAO, 2013).

De acverdo con la norma oficial mexicana NOM-247-SSA 1-2008, los tipos de pan que existen son el blanco, de harina integral, dulce, pasta, pastel o panqué y pay, que es el producto elaborado con harina de cereales o galleta molida, azúcares, agua y sal, con o sin leudante, grasas o aceites comestibles, fruta, crema pastelera, ingredientes opcionales y aditivos para alimentos, moldeado en forma de corteza para contener un relleno dulce o salado; puede ser cubierto horneado, frito o congelado.

La elaboración de un pay de harina de frijol negro San Luis (Phaseolus vulgaris L.) es razonable desde el punto de vista económico y social para los productores (Juárez-lbarra, 2011; Longoria-Cordero, \& Rivera-Acosta, 2015), principalmente para el estado de Zacatecas, primer lugar como productor de esta leguminosa porque además de dar un valor agregado y obtener mayores ganancias, se ofrece un producto con alto nivel nutrimental y beneficios para la salud.

\section{MATERIALES Y MÉTODOS}

Esta investigación se llevó a cabo con base en la norma oficial mexicana NOM-247-SSA1-2008 en el taller de alimentos del Instituto Tecnológico Superior Zacatecas Norte (ITSZN) y en la panificadora La Estrella, empresa comercial propiedad de la familia García Castañeda, ubicada en el municipio de Río Grande, Zacatecas, México. El análisis proximal del pay se realizó en el laboratorio de Análisis de Alimentos de la Universidad Autónoma de Zacatecas (UAZ). 


\section{IIVESTIGAGIÓn Y CUERCIA DE LA UกIVERSIDAD AUTÓOOOMA DE AGUASCALIERTES}

\section{Obtención de harina de frijol}

Se llevó a cabo el siguiente procedimiento:

1. Recepción de materia prima.

2. Limpieza del frijol para eliminar impurezas: piedras, polvo y materia extraña.

3. Lavado de grano con agua potable.

4. Secado del grano al sol para eliminar humedad.

5. Molido de grano de frijol en molino eléctrico hasta obtener harina con partículas de 212 micras.

6. Empacado de harina en bolsas de polipapel y almacenamiento a $21^{\circ} \mathrm{C}$. Es recomendable utilizarse lo antes posible para evitar problemas de plagas $u$ hongos.

\section{Elaboración del pay}

La elaboración del pay se realizó de acuerdo al siguiente procedimiento:

1. Recepción de materia prima.

2. Pesado de ingredientes. Se pesaron de forma independiente la harina de trigo y la de frijol. Los demás ingredientes fueron pesados de acuerdo con la fórmula.

3. Mezclado homogéneo de los ingredientes.

4. Amasado homogéneo de la harina.

5. Reposado de la masa durante 30 min dentro del refrigerador, para endurecerla y facilitar su manipulación.

6. Moldeado. Se rellenaron los moldes con la masa.

7. Horneado a $180{ }^{\circ} \mathrm{C}$ durante $5 \mathrm{~min}$. Después se sacó para añadir encima la mermelada de chilacayote. Se reintrodujo al horno y ahí permaneció durante 10 min más.

8. Enfriado a temperatura ambiente.

\section{Determinaciones}

En la evaluación del pay se determinó lo siguiente: las proteínas por el método Kjeldahl, grasas por método Soxhlet de acuerdo a la AOAC (1990), cenizas conforme a la norma mexicana NMX-F-066-S-1978, fibra por el método de digestión NOM-F-90-S-1978, los carbohidratos por diferencia de componentes en el análisis proximal.

\section{Diseño experimental}

Para la elaboración del pay se generaron cuatro combinaciones de harina de trigo y frijol, las mismas fueron: 50-50 (T1), 65-35(T2), 70-30(T3) y 75-25\%(T4), respectivamente, donde la formulación que tuvo las mejores características (olor, color y textura) fue la elegida (pay experimental) para compararla con el pay comercial por medio de una $t$ de Student para ver si existían diferencias significativas $(p<0.05)$ en el análisis proximal.

\section{Análisis estadístico}

El pay experimental se comparó con el comercial desde el punto de vista proximal por medio de $t$ de Student para todas las determinaciones y diagnosticar si existían diferencias significativas ( $p$ $<0.05$ ) mediante los paquetes estadísticos InfoStat (2011), Córdoba, Argentina y Statistica versión 19842000, Tulsa, OK, EE. UU., para la elaboración de gráficos del análisis proximal y el análisis estadístico de los datos.

\section{RESULTADOS}

En la formulación del pay de harina de frijol con mermelada de chilacayote se evaluaron cuatro tratamientos ( $\mathrm{T} 1, \mathrm{~T} 2, \mathrm{~T} 3$ y $\mathrm{T} 4)$, de los cuales se decidió descartar los T1, T2 y T3, debido a que no tenían la apariencia y textura deseada. Por ello se tomó en cuenta sólo el T4; que sí cumplió con las características deseadas en cuanto a textura, sabor y color. A su vez, se comparó con el pay comercial en contenido proximal.

El contenido de proteína fue de $6.3 \%$ para el pay experimental por $2.4 \%$ en el comercial, lo que significa que tiene más del doble de contenido de proteína. Esto pudo deberse al tipo de fuente de origen del nutrimento; también se compararon estadísticamente y se observaron diferencias significativas $(p<0.05)$. Esto puede encontrarse en la figura 1.

En cuanto al contenido de grasa para el pay experimental se obtuvo un valor de $20.0 \pm 0.3$ por cada $100 \mathrm{~g}$ de muestra; por el contrario, la muestra comercial tuvo $17.3 \pm 0.1$, esto significa que el contenido es mayor en el pay experimental; sin embargo, la grasa es vegetal y en la comercial $53.7 \%$ es grasa saturada, lo que puede ocasionar un mayor nivel de colesterol en el consumidor, al contrario del pay experimental. También se compararon estadísticamente y se observaron diferencias significativas ( $p<0.05$ ), lo cual se observa en la figura 2.

En lo que respecta al contenido de carbohidratos para el pay experimental, el valor fue de $61.8 \pm 0.2 \mathrm{~g}$ por cada $100 \mathrm{~g}$ de muestra; por otra parte, la muestra comercial tuvo $54.2 \pm 0.4 \mathrm{~g}$. Esto 
IIVESTIGACIÓn Y CIERCIA DE LA UNIVERSIDAD AUTÓNOMA DE RGUASCALIERTES

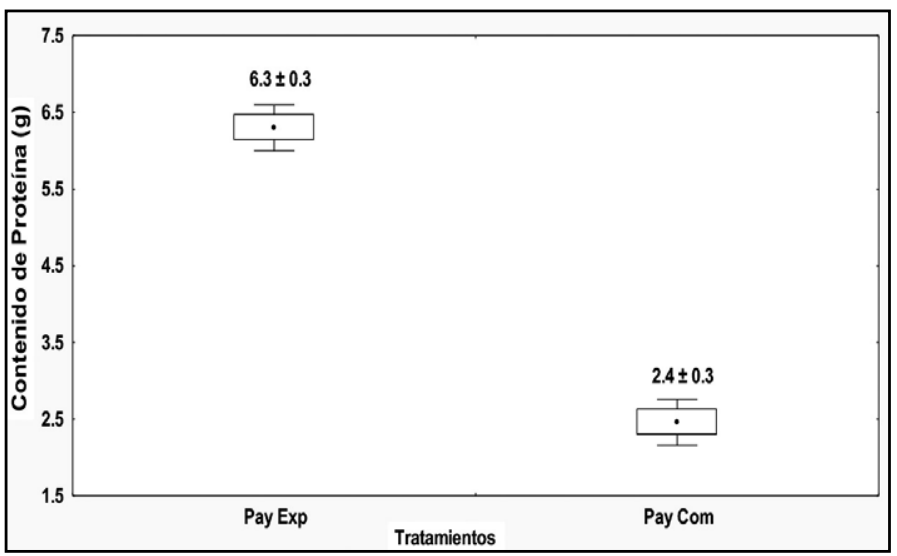

Figura 1. Comparación entre el contenido de proteína del pay experimental y el comercial.

Elaboración propia.

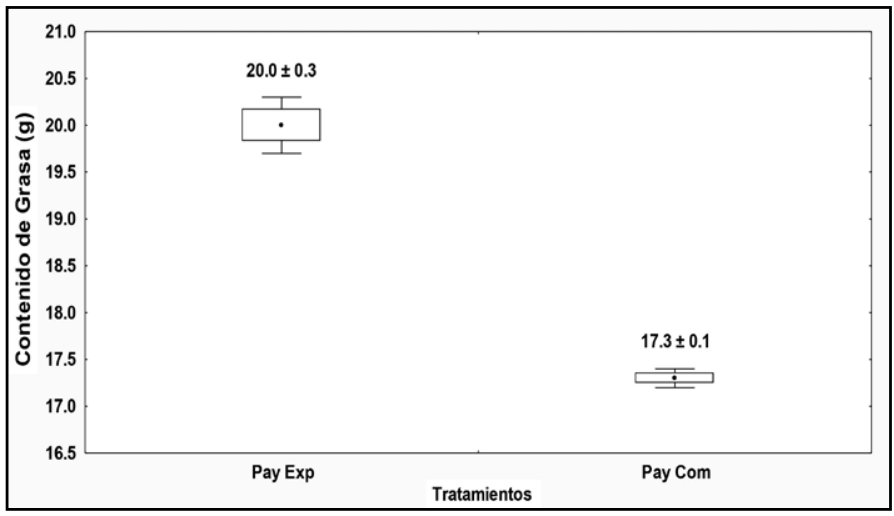

Figura 2. Comparación entre el contenido de grasas del pay experimental y del comercial.

Elaboración propia.

significa $12.3 \%$ más en el experimental. Sin embargo, se encuentra en los límites permitidos (NOM-247SSA 1-2008). Como puede observarse en la figura 3 , se realizó una comparación estadística entre ambos tratamientos y se encontraron diferencias significativas $(p<0.05)$.

En el contenido de fibra, el pay experimental presentó $0.9 \%$ contra $0.3 \%$ del pay comercial, esto significa que es tres veces mayor. La OMS (2015) recomienda una ingesta de 25 a $35 \mathrm{~g} / \mathrm{d}$ en mayores de 18 años y de $0.5 / \mathrm{kg}$ en menores de 10 años; por ello, este pay cubriría de 2.6 a $3.6 \%$ de los requerimientos diarios. Asimismo, se realizó una comparación estadística entre ambos preparados y se encontraron diferencias significativas $(p<0.05)$ en este componente. Esta información puede corroborarse en la figura 4.

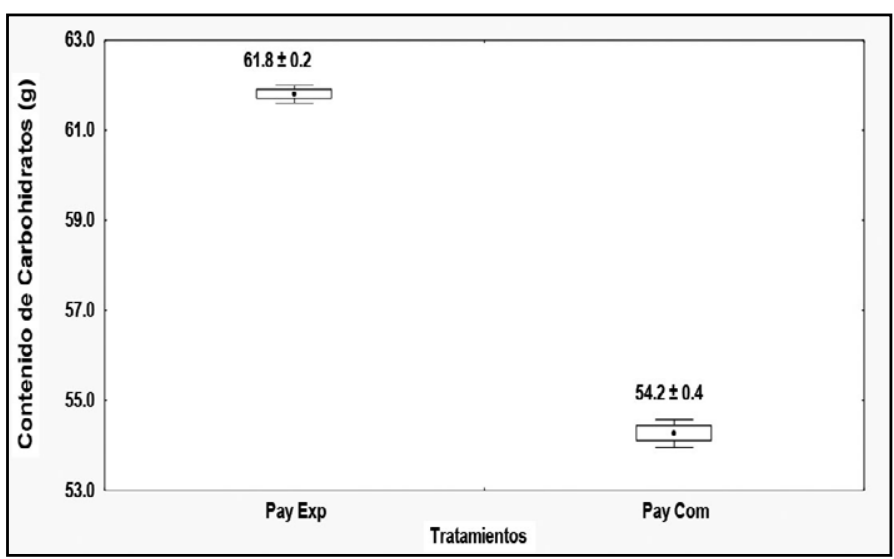

Figura 3. Comparación entre el contenido de carbohidratos del pay experimental y el comercial.

Elaboración propia.

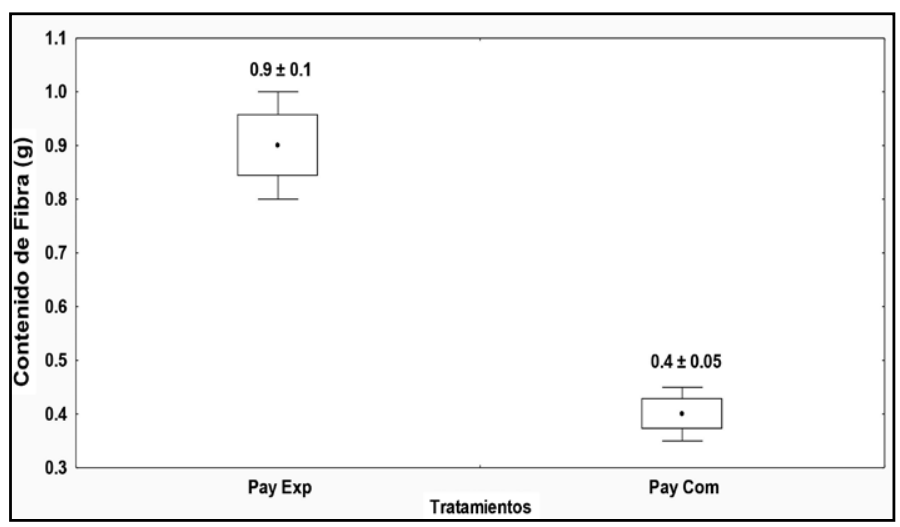

Figura 4. Comparación entre el contenido de fibra del pay experimental y el comercial.

Elaboración propia.

DISCUSIÓN

\section{Proteína}

León Marrou y Villacorta González (2011) realizaron un trabajo con sustitución parcial de harina de trigo (Triticum aestivum) por arracacha (Arracacia xanthorrhiza Bancroft) fortificada, en el cual obtuvieron $8.3 \%$ de proteína. Los autores determinaron con base en el análisis estadístico de las características físicas y prueba de preferencia. Mousa, AlMohizea y Al-Kanhal (1992) compararon la composición química y el valor nutritivo de seis tipos de panes de consumo local en Arabia Saudita (samouli, mafrood, burr, tannouri, tamees y korsan) elaborados con harina de trigo. Los contenidos de proteína oscilaron entre $12.2 \%$ (samouli) y $13.8 \%$ (korsan). 


\section{IIVESTIGAGIÓn Y CUERCIA DE LA UNIVERSIDAD AUTÓnOMF DE RGUASCALIETTES}

Por otra parte, la FAO (2013) menciona que las necesidades de proteínas recomendadas para adultos de 18 años en adelante son de $1 . \mathrm{g} / \mathrm{kg} / \mathrm{d}$; es decir, si tienen un peso promedio de $65 \mathrm{~kg}$ necesitan consumir $65 \mathrm{~g}$ de proteína al día. Asimismo, Serrano y Goñi (2004) mencionan que el requerimiento diario de proteína es de 46-50 g para las mujeres (15-50 años) y de 63 g para los hombres (15-50) años.

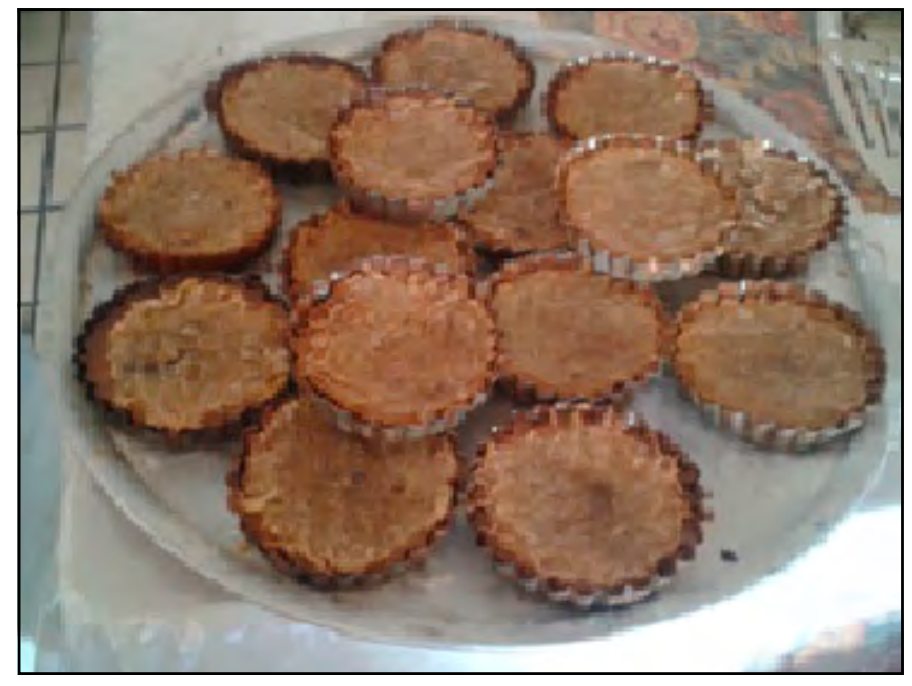

Figura 5. Pay de frijol con mermelada de chilacayote. Fotografía tomada por los autores.

\section{Grasa}

En la investigación que realizaron León Marrou y Villacorta González (2011) obtuvieron $10.1 \%$ de grasa. Mousa et al. (1992) compararon la composición química y el valor nutritivo de diferentes tipos de panes de consumo local en Arabia Saudita, mencionados anteriormente. En los mismos, el contenido de grasa fluctuó entre $0.6 \%$ (burr) y $3.3 \%$ (tamees).

\section{Carbohidratos}

En el trabajo de León Marrou y Villacorta González (2011) se obtuvieron los siguientes resultados: $55.1 \%$ de carbohidratos con valor energético de $344.8 \mathrm{kcal} / 100$ g. Por otra parte, Mousa et al. (1992) obtuvieron una aportación de $70.8 \%$ (Korsan) y $82.3 \%$ (mafrood) de carbohidratos. De la misma manera, la FAO (2013) y Pickut (2013) mencionan que la cantidad de carbohidratos que debe incluir la alimentación de cada persona se estima como un porcentaje del total de calorías. Varían entre 45 y $65 \%$ del aporte calórico total. Por ejemplo, si se consumen alrededor

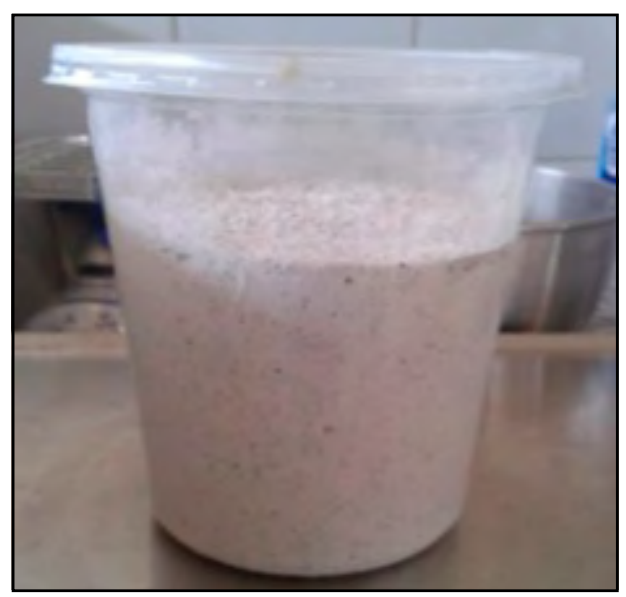

Figura 6. Harina de frijol. Fotografía tomada por los autores.

de 2,300 calorías en el día, se deben obtener entre 1,035 y 1,495 calorías de carbohidratos, esto es igual a 259 g y $374 \mathrm{~g}$ de carbohidratos (Galván-Moreno, \& Sagahón-Canales, 2014; Juárez-lbarra, 2011 ; MárquezRodríguez, \& López-Aguilar, 2014).

\section{Fibra}

Si se retoma la investigación de León Marrou y Villacorta González (2011), se tiene que el contenido de fibra por cada $100 \mathrm{~g}$ es de 2.7g. La FAO (2013) menciona que por cada $100 \mathrm{~g}$ de pan común se encuentra un total de $3.7 \%$ de fibra, en pan integral $6.9 \%$, en pan de salvado de avena $13.5 \%$, mientras que para el pan de salvado de trigo hay $44.5 \%$ de este componente.

De acuerdo con el Comité de Expertos FAO/ OMS la recomendación diaria de fibra dietética total para adultos es de $25 \mathrm{~g} / \mathrm{d}$. Los expertos en nutrición recientemente han publicado una recomendación de consumo para adultos de 19-50 años, la cual es de $30 \mathrm{~g}$ para hombres y de $35 \mathrm{~g}$ para mujeres (Juárez-lbarra, 2011). Datos preliminares de la Encuesta Nacional de Nutrición (ENSANUT) de 2012 (Terrones, 2013) revelaron un consumo de fibra en niños de etapa escolar de 14.1 g. Esta cifra no representa ningún aumento comparado con el reportado de $14.0 \mathrm{~g}$ de consumo por día. Los autores de este trabajo infieren que los problemas recurrentes de estreñimiento en la edad infantil pueden ser resultado del insuficiente consumo de fibra. 
IIVESTIGACIÓn Y CIERCIA DE LA UחIVERSIDAD AUTÓNOMH DE RGUASCALIERTES harina de frijol (figura 6) en este tipo de productos. Esto contribuirá a generar nuevas alternativas y valor agregado para los productores y en especial para los productores de frijol negro.
Se obtuvo un pay con más del doble de contenido en proteína y tres veces más fibra que el pay comercial (figura 5), en esto radica la importancia y uso de

\section{REFERENCIAS}

- Ayala-Garay, A. V., Schwentesius Rindermann, R. E., \& Almaguer-Vargas, G. (2008). La competitividad del fríjol en México. El Cotidiano, 147, 81-89.

- Bello Pérez, S. (2006). El uso de fríjol común (Phaseolus vulgaris) como planta medicinal. Tesina del Diplomado Medicina tradicional de México y sus plantas medicinales. TlahuiMedic. Recuperado el 30 de abril de 2010, de http://www. tlahui.com/medic/medic21/frijol.htm

- Champ, M. (2002). Grain legumes and health. A workshop in 2001. Grain Legum, 35, 13-14.

- EcuRed. Conocimiento con todos y para todos (2015). Frijol. [Serie en red]. Recuperado en octubre de 2015, de http:// www.ecured.cu/index.php/Frijol

- Fideicomisos Instituidos en Relación con la Agricultura. (2015). Panorama Agroalimentario. Frijol 2015 [Serie en red]. Recuperado en junio de 2016, de https://www.gob.mx/cms/ uploads/attachment/file/61950/Panorama_Agroalimentario_ Frijol_2015.pdf

- Flórez, A. (2007). Efectos genéticos, ambientales y de interacción sobre componentes químicos relacionados con el valor sensorial de las judías grano (Phaseolus vulgaris L.). Tesis de doctorado. España: Universitat Politécnica de Catalunya.

- Galván-Moreno, M. J., \& Sagahón-Canales, E. C. (2014). Desarrollo de un pan tipo ranchero de harina de frijol (Phaseolus vulgaris L.) de la variedad negro San Luis con alto nivel nutrimental y aceptable sensorialmente. Tesis profesional. Zacatecas, México: Instituto Tecnológico Superior Zacatecas Norte.

- Gálvez, A., \& Salinas, G. (2015). El papel del frijol en la salud nutrimental de la población mexicana. Revista Digital Universitaria, 16(2). Recuperado de http://www.revista.unam. $\mathrm{mx} / \mathrm{vol} .16 / \mathrm{num} 2 / \mathrm{art} 12$ /

- Granito, M., Guinand, J., Pérez, D., \& Pérez, S. (2009). Valor nutricional y propiedades funcionales de Phaseolus vulgaris procesada: un ingrediente potencial para alimentos. Interciencia, 34(1), 64-70.

- Juárez-lbarra, C. A. (2011). Desarrollo de una barra de frijol (Phaseolus vulgaris L.) con propiedades nutritivas y compuestos fitoquímicos. Tesis profesional. Zacatecas, México: Instituto Tecnológico Superior Zacatecas Norte.

- León Marrou, M. E., \& Villacorta González, M. Y. (2011). Valor nutritivo de pan con sustitución parcial de harina de trigo (Triticum aestivum) por arracacha (Arracacia xanthorrhiza Bancroft), fortificado. Revista Venezolana de Ciencia y Tecnología de Alimentos, 1 (2), 244-261.

- Longoria-Cordero, M., \& Rivera-Acosta, M. (2015). Desarrollo de una barra nutritiva de nopal, avena y frijol con relleno de mermelada de manzana de aceptables características sensoriales y alto contenido proteico y fibra. Tesis profesional. Zacatecas, México: Instituto Tecnológico Superior Zacatecas Norte.

- Márquez-Rodríguez, M., \& López-Aguilar, N. (2014). Desarrollo de una barra de maíz, frijol, amaranto y miel con altas propiedades nutricionales y aceptables características sensoriales. Tesis profesional. Zacatecas, México: Instituto Tecnológico Superior Zacatecas Norte.

- Mousa, E. I., Al-Mohizea, I. S., \& Al-Kanhal, M. A. (1992). Chemical composition and nutritive value of various breads in Saudi Arabia. Food Chemistry, 43(4), 259-264.

- Official Methods of Analysis. (1990). Procedimiento para determinar materia grasa. Método Soxhlet (15a. ed.). EE. UU.: AOAC.

- Organización de las Naciones Unidas para la Alimentación y la Agricultura. (2013). Necesidades nutricionales [Serie en red]. Recuperado en mayo de 2015, de www.fao.org/docrep/014/ am401s/am401s03.pdf

- Paredes-C., M., Becerra-V., V., \& Tay-U., J. (2009). Inorganic nutritional composition of common bean (Phaseolus vulgaris L.) genotypes race Chile. Chilen Journal of Agricultural Research, 69(4), 486-495.

- Pickut, W. (2013). La ingesta diaria recomendada de calorías, carbohidratos, grasas, sodio y proteínas [Entrada en blog]. EHow en español. Recuperado en abril de 2014, de http:// www.ehowenespanol.com/ingesta-diaria-recomendadacalorias-carbohidratos-grasas-sodio-proteinas-sobre_128051/

- Rodiño, P. (2000). Estudio componentes químicos de las judías. Food Biochemistry, 18, 297-309. 


\section{IIVESTIGAGIÓn Y CUERCIA DE LA UחIVERSIDAD AUTÓNOMA DE AGUASCALIERTES}

- Secretaría de Agricultura, Ganadería, Desarrollo Rural, Pesca y Alimentación. (2010). Frijol. Recuperado el 12 de abril de 2010, de http://www.sagarpa.gob.mx/vl/subagri/info/sp/ frijol/prgto.pdf

- Secretaría de Salubridad y Asistencia. (1978). Norma Mexicana NMX-F-066-S-1978. Determinación de cenizas en alimentos. Diario Oficial de la Federación, 3 de noviembre de 1978. Recuperado de http://www.colpos.mx/bancodenormas/ nmexicanas/NMX-F-066-S-1978.PDF (1979). Norma Oficial Mexicana NOM-F-90-S-1978. Determinación de fibra cruda en alimentos. Diario Oficial de la Federación, 27 de marzo de 1979. Recuperado de http:// www.dof.gob.mx/nota_detalle.php? codigo $=4799842 \&$ fec $\mathrm{ha}=27 / 03 / 1979$

- Secretaría de Salud. (2009). Norma Oficial Mexicana NOM247-SSA 1-2008. Productos y servicios. Cereales y sus productos. Cereales, harinas de cereales, sémolas o semolinas. Alimentos a base de: cereales, semillas 62 comestibles, de harinas, sémolas o semolinas o sus mezclas. Productos de panificación. Disposiciones y especificaciones sanitarias y nutrimentales. Métodos de prueba. Diario Oficial de la Federación, 27 de julio de 2009. Recuperado en febrero de 2015, de dof.gob. $\mathrm{mx} /$ nota_detalle.php?.codigo $=5100356 \&$ fecha $=27 / 07 / 2009$

- Serrano, J., \& Goñi, I. (2004). Papel del fríjol negro Phaseolus vulgaris en el estado nutricional de la población guatemalteca. Archivos Latinoamericanos de Nutrición, 54(1), 36-44.

- Servicio de Información Agroalimentaria y Pesquera. (2015). Cierre de la producción agrícola por estado [Serie en red]. Recuperado en octubre de 2015, de http://www.siap.gob. $\mathrm{mx} /$ cierre-de-la-produccion-agricola-por-estado/

- Terrones, M. (21 de junio de 2013). La fibra en el ciclo de la vida [Entrada en blog]. Forbes México. Gourmet. Recuperado en junio de 2014, de http://www.forbes.com.mx/sites/la-fibra-enel-ciclo-de-la-vida/

- Ulloa, J. A., Rosas Ulloa, P., Ramírez Ramírez, J. C., \& Ulloa Rangel, B. E. (2011). El frijol (Phaseoulus vulgaris): su importancia nutricional y como fuente de fitoquímicos. Revista Fuente, 3(8), 5-9.

- Voysest, O. (2000). Mejoramiento genético de frijol (Phaseolus vulgaris L.): legado de variedades de América Latina 19301999. Publicación 321. Cali, Colombia: CIAT. 